\section{Las universidades públicas mexicanas en la era del conocimiento. Procesos de cambio a cien años de la reforma de Córdoba}

Ernesto Treviño Ronzón

\section{Resumen}

El propósito de este escrito es abordar algunas de los principales rasgos en materia de políticas educativas de nivel superior en México, tomando como referencia algunos procesos globales recientes enmarcados en las nuevas narrativas del conocimiento. En la discusión se toma como referencia la relevancia de algunos preceptos emanados del movimiento de Reforma Universitaria de 1918 y sus eventuales ecos en diferentes partes de América Latina y en México. El escrito tiene una orientación conceptual, pero en su desarrollo se da referencia de algunos documentos y propuestas de política que sirven para dar sustento a las afirmaciones. El principal argumento a desarrollar es que las universidades públicas de México, como otras de América Latina se están de transformando en entidades híbridas que experimentan efectos de despolitización constantes, como resultado del intento por responder a los preceptos de autonomía de gobierno y gestión, a las políticas de los gobiernos locales y nacionales, y recomendaciones de agentes externos quienes, sin tener poder formal, poco a poco han introducido nuevas racionalidades que hacen sistema con discursos de igualdad, equidad y responsabilidad social.

\section{The Mexican Public Universities in the Knowledge Era. Processes} of Change one hundred years after the Cordoba Reform

\footnotetext{
Abstract

The purpose of this paper is to address some of the main features of higher education policy in Mexico, taking as reference some recent global processes framed in the new knowledge narratives. In the discussion, the relevance of some precepts emanating from the university reform movement of 1918 and its eventual echoes in different parts of Latin America and Mexico are taken as reference. The paper has a conceptual
}

\section{Palabras clave:}

educación superior, universidad pública, sociedad del conocimiento, Reforma.
Keywords:

higher education, public university, knowledge Society, Reform. 
1. El proceso de reforma iniciado hace más de cien años y sus consecuencias, sus alcances y limitantes, es poco conocido entre los especialistas o investigadores educativos de generaciones recientes en México. Pocos de los especialistas en educación superior han profundizado en él como lo hicieron los estudiosos del sistema de educación superior mexicano en la década de 1970 y

1980. Esto ocurre también en el caso de otros movimientos como el de autonomía universitaria en México o el movimiento estudiantil de 1968.

Las causas de suerte de ausencia deben ser objeto de atención urgente. orientation but in its development, reference is given to some documents and policy proposals that serve to support the affirmations. The main argument to be developed is that public universities in Mexico, like others in Latin America, are transforming themselves into hybrid entities that experience constant depoliticization effects, as a result of the attempt to respond to the precepts of governance and management autonomy, policies of local and national governments, and recommendations from external agents who, without having formal power, have little by little introduced new rationalities that have been articulated with discourses of equality, equity and social responsibility.

\section{Presentación}

El objetivo de este escrito es abordar algunas de las dinámicas de transformación de las universidades públicas en México tomando como referencia procesos globales recientes en materia de políticas educativas, producidos en la trama discursiva de la sociedad y la economía del conocimiento. La discusión se enmarca en una reflexión de carácter situado sobre la vigencia de algunos preceptos emanados del movimiento de Reforma Universitaria de 1918 en Córdoba, y sus eventuales ecos en México.

Me parece que es una discusión necesaria pues las universidades públicas en México, como las de otras partes del mundo están atravesadas y tensionadas por múltiples procesos que ponen en colisión las convicciones más elementales y por muchos años defendidas, como la de autonomía o libertad de cátedra, con otras que parecen exógenas, como las de calidad o competitividad. Las ideas aquí desarrolladas tienen una orientación primordialmente conceptual y toman como referente algunas de las herencias políticas que se han diseminado por el pensamiento universitario desde hace más de cien años, a partir del proceso de reforma universitaria iniciado en Córdoba y que entre muchos especialistas es un referente obligado para entender el devenir de las universidades públicas en América Latina. ${ }^{1}$

Sin duda, las universidades públicas mexicanas, en particular las que surgieron hacia la mitad del siglo XX son herederas de una gran cantidad de movimientos políticos e intelectuales algunos de carácter "revolucionario" y otros más bien "conservadores". Entre ellos está incluido, por supuesto, la así llamada Reforma Universitaria de 1918. Hoy en día, es posible establecer los vínculos entre el diseño institucional, las formas de gobierno y las estructuras organizacionales internas de varias universidades con algunos de los postulados de la reforma, sus consecuencias y reapropiaciones en forma de demandas y procesos de autonomía, de popularización, de masificación y de democratización. No se trata, por supuesto, de afirmar que podamos al pie de la letra y con ánimo verificacionista rastrear dicha relación, pues es algo poco útil y posiblemente sería pobremente ilustrativo de los vínculos. En cambio, se trata de pensar que a cien años de la reforma hay un cierto resto, una herencia que se ha diseminado (Derrida, 1997) y que es necesario revisitar ante una serie de cambios que desde hace un par de décadas están modificando radicalmente la cara de las instituciones de educación superior.

El principal argumento a desarrollar es que las universidades públicas de México, como otras de América Latina se están transformando en entidades híbridas, en la tensión de responder a los preceptos de autonomía de gobierno y gestión, la presión de los gobiernos locales y las tendencias impulsadas por agentes externos que, sin tener poder formal, poco a poco han introducido nuevas racionalidades que hacen sistema con discursos de igualdad, equidad y responsabilidad social. Este hibridismo tiene diferentes efectos despolitizantes que deben ser sometidos a nuevos y diversos procesos de revisión, de desmontaje y crítica, pues pueden tener consecuencias todavía más drásticas para la idea de universidad pública de orientación científica y de compromiso social que se ha intentado construir durante varias décadas. 
Como ya se ha indicado, el escrito tiene una orientación conceptual, pero en su desarrollo se dan referencias de documentos y propuestas de política que sirven para dar sustento a las afirmaciones vertidas. Para desarrollar la discusión se hará referencia la Universidad Veracruzana (UV), una de las universidades públicas más grandes de México y que puede funcionar como ejemplo para ilustrar la discusión, y se echará mano de nociones conceptuales como la de reforma, desacuerdo e imaginario, las cuales serán usadas en diferentes momentos del escrito.

El escrito se organiza de la siguiente manera. Primeramente, se abordarán algunos rasgos del sistema de educación superior en México y particularmente del así llamado subsistema de universidades públicas. En esta revisión se hará referencia demás a algunos de los ecos que la reforma de Córdoba ha dejado en dicho sistema. En un segundo momento, se abordará la narrativa de la sociedad del conocimiento con énfasis en el peso que ha tenido en la definición de las tareas de docencia, investigación y vinculación. En la tercera sección se desarrollará una discusión sobre cómo los procesos de reforma han llevado de dinámicas de politización a otras de despolitización en años recientes, conduciendo a la emergencia de un paradigma de gestión protogerencialista que debe ser sometido a estrés y en su caso, desmontado dada su agresividad y sus consecuencias sociales, educativas y políticas.

\section{Las universidades públicas en México y los ecos de la Reforma Universitaria}

El sistema educativo mexicano es uno de los más grandes y diversificados de América Latina, aunque no siempre lo ha sido así. Como en otras partes del mundo, como en la Argentina de 1918, la educación superior de México, nació siendo un ámbito muy acotado, para élites intelectuales, políticas y religiosas, centralizado y dependiente de los principales poderes del Estado Mexicano. La presencia de sectores populares, campesinos, indígenas o mujeres en las aulas universitarias era muy elemental cuando no prácticamente inexistente. El proceso de cambio inició siguiendo parcialmente el proceso inaugurado por la reforma de 1918 en Córdoba y replicado de diferentes formas en diferentes partes de América Latina.

En buena medida, y como veremos a continuación, es posible señalar que el cambio del sistema educativo de nivel superior y en particular de tipo universitario en México es heredero de algunos de los principales preceptos de la reforma de Córdoba pues se ha orientado a una búsqueda permanente de autonomía de gobierno, libertad de cátedra, de apertura a los diferentes sectores, de orientación científica y procurando el involucramiento de los estudiantes en el gobierno universitario. ${ }^{2}$ Aunque es pertinente señalar que la búsqueda de estos valores está desigualmente distribuida por el tiempo y tiene variaciones significativas dado el contexto local y regional.

Posiblemente la expresión más visible del proceso de cambio heredero de la reforma fue el movimiento estudiantil y social que, con una huelga de promedio y diversas peticiones, culminó en la publicación de la Ley Orgánica de la Universidad Nacional Autónoma de México (UNAM) (Marsiske, 1999; 2015). Esto no solo marcó un punto de inflexión en la historia de la educación superior mexicana, sino que también generó un impacto mayúsculo en la historia de la educación pública del país. Es necesario reconocer aquí que, si bien esta Ley es la que crea la UNAM como propiamente la conocemos, previo a este significativo evento otras universidades obtuvieron su autonomía como lo son la Universidad Michoacana de San Nicolás de Hidalgo en 1917 y la Universidad Autónoma de San Luis Potosí en 1923. Esta fue de hecho, la primera en apropiarse de manera evidente de los preceptos de Córdoba.
2. Los trabajos que resumen los preceptos de la Reforma Universitaria de 1918 se han incrementado en los últimos años de manera impresionante. Algunos se han enfocado en reconstruir su contexto de emergencia, otros las demandas originales, otros los alcances y otros sus consecuencias educativas, sociales y en su caso políticas. Al respecto pueden leerse trabajos como los de Buchbinder (2018), Saur y Servetto (2013), Tünnermann (2008). 
3. En el 2017 se cumplieron cien años de la promulgación de la Constitución de 1917. El aniversario motivó diversos análisis, tanto sobre las condiciones de gestación y promulgación del documento, como sobre sus alcances, sus limitaciones, y por supuesto sus transformaciones en el tiempo. En el proceso de análisis asuntos como la educación han sido objeto de diferentes discusiones, pero paradójicamente la educación superior es la que menos atención recibió.

Esto se puede deber a la estabilidad de las políticas en el sector, asunto que se tratará más adelante. Para una revisión del tema educativo

puede verse el trabajo coordinado por Treviño, Galindo y Ducey (2017).
$\mathrm{Al}$ respecto no está de más recordar que doce años antes de 1929, se había promulgado la nueva constitución de México, conocida como la Constitución de 1917, que actualizaba una serie de acuerdos colectivos sobre las responsabilidades del Estado nacional. La Constitución fue en un sentido, el resultado y proceso de cierre del ciclo revolucionario iniciado en 1910 y para no pocos, con ella, la fracción ganadora de la revolución estaba tratando de "poner en un baúl" lo que el gobierno de Porfirio Díaz -en el poder por más de treinta años- había dejado a su partida. Esto sería una exageración, pues aunque la Constitución de 1917 estuvo claramente influenciada por la perspectiva del gobierno del presidente Venustiano Carranza, en la confección del texto se contó con la participación de diferentes actores políticos de diferentes posiciones, de todos los estados de la república y tomó forma en un marco de atención y debate que había iniciado previamente en los congresos locales de los estados de la república (Marván, 2017) y en los cuales el tema de la educación era protagónico.

Ahora bien, a pesar del énfasis garantista y de derechos sociales expresado en la Constitución de 1917, es claro que su compromiso con la educación pública estaba acotado al ciclo básico. Por ello, el crecimiento poblacional aunado a una gran cantidad de cambios locales y regionales en diferentes órdenes de la vida nacional pronto hicieron evidente que el marco de regulación y el compromiso público con la educación universitaria estaba siendo muy acotado. De tal forma que el movimiento que culminó en la Ley de Autonomía Universitaria de 1929 y las posteriores, puede ser entendido al mismo tiempo como una demanda y una actualización de los compromisos del ciclo revolucionario con la educación, pero ya no en manos solamente de políticos, intelectuales o gobernantes. ${ }^{3}$

Algo que rara vez se enfatiza, es que estos eventos en la vida de las universidades de México dan cuenta de un proceso de politización, de emergencia de ciertas formas de lo político que interrumpen e instalan una forma de litigio que, al mismo tiempo, interrumpe el flujo de la discursividad predominante (Rancière, 1996). Esta forma de politización no pasó necesariamente por las formas tradicionales de asociación política, como las sindicales o las partidistas, sino que se configuró en forma de asociaciones o agrupaciones de estudiantes que, sin duda, se articularon eventualmente con el poder político formal, pero que no necesariamente fueron absorbidos por ellos.

La transformación de la educación superior en México ha observado procesos contrapuestos, por un lado, ha sido estable y básicamente incrementalista, es decir, agregadora de decisiones. Y en otros momentos pareció introducir variaciones significativas. Por ejemplo, el peso del socialismo se dejó sentir cuando el General Lázaro Cárdenas, presidente electro para el periodo 1934-1940 se declaró un convencido de la educación superior técnica y tecnológica y de su potencial impacto positivo en el desarrollo de México. Siguiendo esa convicción, creó el Instituto Politécnico Nacional, maximizando las experiencias de varias instituciones ya vigentes en México y como una alternativa a la UNAM, que en su perspectiva respondía más a las aficiones intelectuales de ciertas élites sociales y culturales.

Después de aquel momento decisivo se observó un proceso de apertura gradual de universidades públicas estatales y federales, y también de institutos tecnológicos, si bien en condiciones de limitaciones económicas y de muchas variaciones en las orientaciones pedagógicas y curriculares. En esa época, de la década de 1940 a finales de la década de 1960, las universidades públicas nuevas y añejas siguieron en su mayoría respondiendo a dinámicas de centralización regional y política. Por ejemplo, la Universidad Veracruzana, una de las cinco universidades más grandes de México se creó en 1944 y se localizó en la ciudad de Xalapa, Veracruz, a partir de un pequeño número de escuelas de nivel superior. No nació autónoma, y no lo sería hasta mediados de la década de 1990. Durante su primera etapa de vida estuvo fuertemente sometida a las decisiones de los 
gobiernos locales y esta relación ha cambiado, pero no del todo, aun siendo autónoma. Este es el caso para muchas universidades ubicadas en otros estados de la república. ${ }^{4}$

Si bien más estudiantes de sectores populares siguieron ingresando, también se mantuvo la ubicación de las instituciones educativas en los grandes centros urbanos y la asociación de la educación al desarrollo del país, aunque de una manera más declarativa que concreta. En ese entonces se observaron momentos de distanciamiento y enfrentamiento entre el sector universitario y las autoridades estatales y federales, cuyo punto más conocido es el movimiento de 1968 y la matanza de Tlatelolco, aunque, por supuesto, hubo otros movimientos menos visibles en diferentes partes del país.

El ciclo o etapa de la así llamada expansión de la educación nacional se puede ubicar con relativa claridad a mediados de la década de 1970, se acentuó en la década de 1980 y eventualmente dio paso al ciclo de "diversificación" de la década de los años noventa. El ciclo de expansión incluye varios procesos, contrapuestos y problemáticos: incremento de la matrícula en licenciatura y posgrado, apertura de diversas instituciones de educación superior -incluidas universidades e institutos tecnológicos-, creación de nueva legislación y de muy visibles universidades públicas y centros de investigación, baja profesionalización del profesorado, crisis económicas, financiamientos limitados y deterioro de la carrera académica. ${ }^{5}$ Aun así, en esta época la cantidad de estudiantes universitarios creció a un ritmo acelerado y hacia el final de esa época, junto con la llegada de los procesos de globalización inició el ciclo de diversificación que, bajo la luz de políticas neoliberales dio forma al sistema educativo como lo conocemos ahora (Rodríguez, 1995).

A inicios de la década de 1990 México ingresó a la Organización para la Cooperación y el Desarrollo Económicos y firmó un tratado de libre comercio con Canadá y Estados Unidos de Norteamérica, creando el mercado integrado más grande del mundo hasta ese momento. Estos acuerdos pusieron mucha presión en diversos sectores del país, incluido el educativo que comenzó a ser observado con los parámetros de países europeos y de los vecinos del norte. También fue asumido como un sector susceptible de ser dirigido como un ámbito estratégico de la economía, pero no como lo postulaba el modelo desarrollista de mediados de siglo e inclusive distante del modelo de capital humano de la década de 1960.

Como resultado de lo ocurrido en esa época, actualmente, el sistema de educación superior se compone por universidades públicas estatales y federales, universidades tecnológicas, politécnicas e interculturales, institutos tecnológicos, universidades a distancia, instituciones educativas para la formación de docentes: universidades pedagógicas y escuelas normales; así como por institutos y centros públicos de investigación.

Asimismo, el sistema de educación superior comenzó a ser crecientemente meritocrático, según los términos de las políticas emergentes durante los años noventa. En esa época se dio la masificación de las evaluaciones para el ingreso de estudiantes, de las evaluaciones para ingresar a la carrera académica y científica, de las evaluaciones como condición para entregar algunos financiamientos científicos y tecnológicos, entre otras dinámicas que en su momento se denominaron "novedosas". Y también se dio el ascenso de la educación superior privada que, si bien tiene una larga historia en México, comenzó a despuntar producto de la liberalización o desregulación de diferentes sectores y de la creciente demanda no atendida, de estudiantes que pretendían ingresar al nivel superior y que el Estado no estaba atendiendo.

Al final de la segunda década del siglo XXI, los niveles educativos disponibles para los estudiantes son los de técnico superior, licenciatura, especialización, maestría y doctorado. Aproximadamente tres millones quinientos mil estudiantes están matriculados
4. La autonomía en las universidades públicas mexicanas incluye la posibilidad de elegir a sus dirigentes, establecer su propia forma de organización, entre otros aspectos. Pero dado que el financiamiento que reciben es de cuatro tipos de fuentes, los lazos con los gobiernos locales y federal son permanentes y no siempre tersos.

5. En la década de 1970 se publica la Ley de Autonomía Universitaria que se va adoptando gradualmente por todo el país (1979). También se crea el Consejo Nacional de Ciencia y Tecnología (Conacyt) (1970), entre otros procesos estratégicos. 
y de ellos cerca del treinta por ciento lo hace en instituciones privadas, el resto en instituciones públicas. Solo tres de cada diez jóvenes en edad de 18 a 22 años, típica para estudiar en el nivel licenciatura tiene un lugar, es decir, la cobertura es de un poco más de treinta por ciento y la oferta todavía está fuertemente concentrada en los grandes centros urbanos del país y los treinta y dos estados de la república, aunque la oferta de algunas modalidades de educación superior ha puesto énfasis en alunas zonas rurales -incluyendo aquí la participación de la educación privada-.

Este panorama tiene muchas lecturas. Por un lado, es indicador de un avance importante en materia de cobertura y diversificación. Por otro, es indicador claro del largo camino que falta por recorrer, pues como es evidente, no solo se observa una muy baja cobertura en relación con la demanda, sino que la respuesta del Estado ha sido muy lenta. Más aun, aunque las universidades públicas estatales son prácticamente en su totalidad autónomas (treinta y dos), la gran mayoría de las instituciones de educación superior públicas de México, donde se incluyen Institutos Tecnológicos, Universidades Federales, Escuelas Normales, Universidades Tecnológicas, entre otras, no lo son, pues dependen en gran medida de las decisiones de los gobiernos para orientar su existencia.

En este sentido, es posible señalar que varios de los preceptos de la reforma de Córdoba han impactado fuerte y positivamente en la vida de las Universidades Públicas en México, alimentando una cierta forma de organización y de gobierno interno. La idea de reforma ha funcionado por mucho tiempo para instalar un marco de representación de lo que es y puede llegar a ser la educación superior universitaria y como lo ha indicado Popkewitz (2007), ha permitido crear nuevas narrativas del pasado, el presente y el futuro. Pero, al mismo tiempo, puesta en el contexto mayor de la educación superior de México, es claro que su alcance es acotado.

\section{El ascenso de la nueva narrativa del conocimiento}

Así, las universidades públicas mexicanas, en la que se centrará la atención ahora, constituyen un entorno diverso, aunque comparten entre sí muchas características organizacionales, políticas y culturales. Uno de los rasgos más interesantes de las universidades es la forma en que viven su autonomía y la forma en que esta se traduce en estructuras políticas, administrativas y de gestión internas las cuales muestran tantos paralelismos como divergencias.

Hasta inicios de la década de 1990 estas estructuras se mantuvieron relativamente estables y hasta cierto punto, anquilosadas, pero la llegada de los procesos de evaluación y de certificación, y de una nueva racionalidad en la entrega de los recursos públicos hacia las instituciones educativas, producto en buena medida de la transformación de la administración pública en el marco de la firma del Tratado de Libre Comercio de América del Norte y del ingreso a la Organización para la Cooperación y el Desarrollo Económicos (OCDE), inició un proceso de cambio o de movilización institucional que en muchos casos no ha concluido interminable.

Las instituciones recibieron el impacto de las nuevas narrativas sobre el conocimiento -la sociedad del conocimiento, la economía del conocimiento-, diseminadas en buena medida por la OCDE, pero también por la Unesco y muchas otras organizaciones internacionales. Los efectos de estas narrativas son en algunos casos evidentes y en otros casos muy sutiles, sin embargo, tienen implicaciones para el ejercicio de la autonomía, para las formas de gobierno, para las dinámicas internas de las universidades y para sus metas institucionales, políticas, educativas, sociales y hasta económicas. Probablemente unas de las más graves consecuencias de estas narrativas son sus gestos despolitizantes. 
¿En qué consisten las narrativas sobre el conocimiento y cómo se articulan con la vida y la misión de las universidades? Llamo nuevas narrativas sobre el conocimiento a aquellas formas de discurso que postulan al conocimiento en sus diferentes expresiones - patentes, credenciales, publicaciones, hallazgos- como la principal fuente de riqueza y motor de las sociedades y de sus economías. Se hicieron visibles en los años noventa, pero su trayectoria se puede trazar hasta la década de 1930, primeramente, desarrolladas en los Estados Unidos de Norte América y luego en Europa y también en diversos países de Asia (Hayek, 1937; Machlup, 1962).

En la década de 1960, académicos como Peter Drucker (1969) desarrollaron teorías acerca de la administración del conocimiento mientras que otros como Alain Tourain (1969), y Jean-Francois Lyotard (1981), entre los años sesenta y setenta desarrollaron advertencias o críticas sobre las nuevas formas de producción y uso del conocimiento que se estaban observando ya en algunas universidades de sus respectivos países.

Aquellas primeras discusiones fueron potenciadas durante la década de los ochentas y los noventas. La irrupción de Internet, la caída del Muro de Berlín y la intensificación de los procesos de globalización, sobre todo de tipo económico, incentivaron estas narrativas en la medida en que nuevos segmentos de la economía comenzaron a depender cada vez más de productos basados en conocimiento, en otras palabras, de los resultados de la investigación básica y aplicada. La agricultura, la medicina, las telecomunicaciones, los productos de consumo, la moda y el entretenimiento, comenzaron a hacer influenciados por la disponibilidad de conocimiento, que, sin embargo, no era producido necesariamente de la manera tradicional. Este nuevo conocimiento, o tipos de conocimiento, implicaba articulaciones diversas entre los innovadores y el mercado, entre la academia y los usuarios, entre las instituciones educativas, los gobiernos y las empresas.

Ante este panorama, diversas organizaciones internacionales (OCDE, 1996; 2000; Unesco, 1998; 2005), así como varios gobiernos nacionales comenzaron a diseñar políticas científicas y de educación superior orientadas a impulsar el desarrollo de una nueva forma de economía basada en el conocimiento y reposicionando el papel de la educación superior en este nuevo marco. Interesantemente este proceso se articuló con la consolidación de un modelo económico neoliberal en distintas partes del mundo, incluido México. También con el ascenso de una nueva narrativa sobre la gestión de los compromisos del Estado que entre otras cosas adoptó una perspectiva de calidad y competitividad que impactó directamente en la forma en que los gobiernos se relacionaban con las instituciones educación superior y ayudaban a que estas cumplieran con sus compromisos sociales. La entrega de recursos públicos en muchos casos comenzó a ser condicionada a la mejora de indicadores, bien en los procesos de selección, bien en los procesos de producción científica, bien en los procesos de certificación o inclusive de vinculación con distintos sectores sociales y económicos.

En la transición del siglo XX al siglo XXI estos procesos se fueron consolidando y los gobiernos nacionales (SEP y CONACYT, 1996), y a partir del año 2000 (SEP, 2001; 2007; 2013), asumieron como una de sus principales metas convertir a México en una sociedad basada en el conocimiento, articulado en torno a una economía del conocimiento altamente integrada en el mercado mundial. Para esto las políticas científicas y educativas debían alinearse a nuevos procesos, a nuevas metas y, en resumen, a un nuevo imaginario de mejora, de calidad y de competitividad (Treviño, 2015).

Es importante tomar en cuenta que muchas universidades, incluida la UV (Universidad Veracruzana, 1998) asumieron rápidamente estas narrativas y las políticas nacionales, traduciéndolas en programas de educación virtual y a distancia, en programas de vinculación, programas y estrategia de mercantilización de algunos servicios universitarios, 
nuevos procesos de internacionalización, nuevos procesos de certificación y evaluación interna. Pero estas acciones no necesariamente se tradujeron en mejores condiciones laborales para los académicos o educativas para los estudiantes.

Al día de hoy la sociedad del conocimiento describe un proceso a través del cual el conocimiento científico se convierte en un motor de diferentes procesos sociales y en particular de la economía (Sther, 2004; Unesco, 2005) y por ello las universidades y otras instituciones de educación superior y de investigación continúan en la búsqueda de productividad científica, de ascenso en los rankings internacionales pero por sobre todo de "visibilidad" y "prestigio". No solo por su función "tradicionalmente asumida" de educar a las personas, sino también y, sobre todo, por su capacidad de responder a las nuevas exigencias de medición y visibilidad nacional e internacional.

Pensar las particularidades de esta articulación es muy importante porque a través de ella se han filtrado una gran cantidad de cambios en las instituciones educativas, algunos de los cuales impactan funciones sustantivas como la docencia, la investigación y la vinculación. Por supuesto, estas responsabilidades siguen vigentes en todas las instituciones, pero se han matizado y, de alguna forma, expresan una tensión interna, pues los cambios no derivan necesariamente de procesos de reflexión interna, sino que responden también a factores externos que se combinan con dinámicas institucionales creando articulaciones problemáticas.

Aquí es donde merece una reflexión puntual las formas de gobierno y la autonomía de las instituciones de educación superior, particularmente de las universidades públicas, pues para muchos académicos y también para numerosas autoridades universitarias, hoy en día se verifica la instalación de un dispositivo de control híbrido. Debido a este dispositivo se deben responder a indicadores nacionales o inclusive internacionales para asegurar financiamientos y respaldos, pero en ocasiones guardan una distancia larguísima con las responsabilidades contextuales y concretas de las universidades públicas. Es el caso de la demanda por publicar en cierto tipo de revistas y editoriales -indexadas en bases de datos e índices "reconocidos", de alto impacto -en los términos de los índices internacionales creados por las casas editoriales que funcionan como empresas transnacionales-, con un cierto ritmo -en los ciclos de evaluación nacional o internacional-, y en temas de "prioridad". Es el caso también de la constante presión para obtener recursos externos para financiar proyectos de investigación de innovación, preferentemente de instancias nacionales e internacionales como suplemento o inclusive como reemplazo de aquello que debería venir del Estado y de las instituciones mismas.

En términos generales es difícil estar en desacuerdo con la importancia de publicar o de tener financiamientos externos. Pero la racionalidad detrás del dispositivo híbrido rebasa por mucho este sentido básico de acuerdo, pues parece instalar una lógica muy diferente, tiene una orientación de mercado, de dirección gerencial -basada en resultados y no en necesidades- y de administración a la distancia -que implica que las personas se autorregulen- (Miller y Rose, 2008); que socava gradual y potentemente la misión social más añeja de las universidades y en más de un sentido, condiciona su existencia.

En este gran contexto es importante tener presente que la nueva narrativa sobre el conocimiento no es una de tipo impositivo, es decir, no "obliga" a nadie, no "excluye" a nadie. Es un tipo de narrativa "inclusiva" compuesta por significantes flotantes con gran capacidad de vaciamiento y, por lo tanto, con una gran capacidad de articulación (Laclau, 2014). Ha sido reapropiada por una importante variedad de actores políticos y económicos, quienes han contribuido a generar diferentes discursos, casi todos seductores y con gran capacidad de convencimiento, pues prometen salvarnos del atraso y de 
la ignorancia, del problema de la pobreza y el desempleo, de los riesgos de la "irrelevancia" de la educación universitaria y de su incapacidad para generar movilidad social.

En cambio, ofrecen un horizonte de plenitud, basado en todas las "virtudes y beneficios" que el conocimiento puede entregar para sociedades educadas y que han aprendido a utilizarlo o explotarlo de maneras innovadoras. Una gran paradoja en este contexto es que las universidades autónomas han decidido recuperar estos planteamientos para orientar sus propios planes y programas de trabajo, lo han hecho precisamente en el marco de su autonomía, de su legítima forma de autogobierno, asumiendo que es la "correcta" forma de conducirse, "la más adecuada", la "socialmente responsable", la "académicamente innovadora", la que asegura su "relevancia" y su "pertenencia".

En este marco la función sustantiva de las instituciones universitarias se mantendría intacta, posiblemente impoluta, pues nadie estaría imponiéndoles ningún tipo de política. Pero como podemos derivar a partir de una revisión documental, tanto la autonomía de gobierno, de decisión y de gestión estarían condicionadas, pues no se trataría de un marco de acción totalmente libre. Por supuesto, desde hace mucho tiempo sabemos que la autonomía siempre es relativa, es decir, no es posible que las universidades estén desconectadas de los gobiernos locales o nacionales, pues al final de cuentas ellos entregan los recursos financieros que de diferentes formas aportamos los ciudadanos y la nación. Pero siempre se ha asumido que las decisiones del gobierno universitario están de alguna forma salvaguardadas por los marcos constitucionales vigentes que dan por supuesto la disponibilidad de recursos financieros.

En los actuales esquemas de política educativa y de financiamiento, dichos recursos tienen un carácter mixto y una parte de ellos derivan de las disposiciones -obligaciones- constitucionales, otros son el resultado de decisiones y esquemas de financiamiento basados en criterios de "competitividad" y de "calidad" que hacen sistema con los discursos de la sociedad y la economía del conocimiento en la versión difundida por varios organismos internacionales y gobiernos nacionales que suscriben sus propuestas $\mathrm{y}$ condicionan la vida y el gobierno de las instituciones.

Pero uno de los mayores efectos de estas dinámicas tiene que ver con lo que llamaré los efectos de despolitización académica y educativa. Hablaré de ellos en la siguiente sección e intentaré relacionarlos con la necesidad de retomar los preceptos de la Reforma de 1918 y de algunas de sus variaciones contextuales, particularmente en el México del siglo XXI.

\section{Los efectos de despolitización en las universidades públicas}

Para muchas personas las reformas educativas pueden tomarse simplemente como un proceso administrativo o de gestión del cambio. En buena medida, esta sería la perspectiva que gobiernos nacionales como los de México, Chile y Brasil adoptaron durante la transición del siglo XX al siglo XXI, al proponerse crear instituciones de calidad, orientadas por indicadores concretos de mejora continua. Sin embargo, más allá de esta componente técnica, la idea de reforma o de transformación, si se quiere, incluye un elemento mucho más interesante, una suerte de simiente o de semilla que contiene aspiraciones, propuestas de diverso tipo y que puede producir efectos deseados y no deseados, e ir muchos más allá del contexto institucional generando incluso un cambio social o político intenso y diverso.

Esta simiente no es simplemente la expresión poliforme de un término, de un significante que resulta atractivo o interesante. Por el contrario, como indicó en su momento 
Derrida (1997), se trata de un término o de un significante que a partir de su puesta en disposición, o de su circulación va generando una serie de significados nuevos, de efectos del sentido en un plano, en un momento unificadores y en otros diversos, en la medida en que se articula con proyectos y políticas, con protestas y aspiraciones, con insatisfacciones y demandas que podrían ser o no gestionadas a través de los instrumentos vigentes, pero que podrían generar también múltiples nuevas necesidades.

La Reforma Universitaria de 1918 generó sin duda un nuevo marco de representación para pensar la universidad pública, su relación con el contexto local, nacional y regional. Pero más allá de eso, inauguró un nuevo marco de politicidad educativa, universitaria y ultimadamente, social. Para no pocos la politicidad derivada de la Reforma fue negativa, pues abrió la posibilidad para que muchas personas se articularán de formas no académicas a la institución universitaria. De hecho, el tipo de movilización derivada de la Reforma es siempre criticada en más de un sentido en diferentes partes del mundo, incluido México, por su énfasis en las formas de gobierno, o en que la participación de los estudiantes en las decisiones institucionales, por encima de la discusión de tipo académico.

Desde mi punto de vista muchas de estas críticas son limitadas, pues la nueva politicidad impactó radicalmente la forma de entender el ejercicio académico, así como la forma de ejercer y de regular la vida en las universidades frente a los paradigmas europeos dominantes a inicios del siglo XX y que eran profundamente excluyentes, clasistas y racistas. Así, desde otro punto de vista, cierto tipo de politización no puede ser negativa puesto que el conocimiento, y la formación de nivel superior no pueden ser vistos como acciones neutrales, libres de debates o de tenciones con el medio social.

Pero a cien años del inicio del ciclo de reforma, y de muchos de los movimientos que derivaron o se articularon con ella, los efectos de politización se han ido diluyendo, se han ido procesando de formas poco promisorias en diferentes universidades públicas y han quedado atrapadas en marcos de gestión. En algunas universidades autónomas, como la Veracruzana, en México, la participación de los estudiantes en la vida institucional está reconocida por su ley orgánica, está codificada y gestionada a través diferentes normatividades. Sin embargo, detiene con gran efectividad la libertad de acción y de propuesta. Los marcos vigentes incorporan a los estudiantes en el gobierno institucional de forma pasiva o subordinada, casi siempre para validar iniciativas elaboradas por autoridades y, en su caso, por académicos; e, interesantemente, esto rara vez es puesto en cuestión.

Ahora bien, es necesario reconocer también que esta participación subordinada no es exclusiva de los estudiantes, pues en el caso del personal académico sobre todo aquellos que no son profesores de tiempo completo, los cuales constituyen la mayoría de la planta docente, la participación en las decisiones estratégicas que forman o derivan en el gobierno de la vida universitaria es muy baja y está condicionada por prácticas y culturas académicas sedimentadas.

Un aspecto clave que las nuevas narrativas sobre el conocimiento han impulsado con gran efectividad es la búsqueda de la excelencia académica en los términos que hemos descrito previamente: publicaciones constantes, proyectos innovadores y financiamientos externos, preferentemente internacionales, profesores con grados de doctor que participan de diferentes asociaciones y cuentan con diferentes reconocimientos. Además, han impulsado una retórica de aseguramiento de los impactos positivos de la investigación y la docencia en los diversos sectores sociales.

Pero esta excelencia académica viene acompañada de un costo político y social, más o menos reconocido en diferentes contextos. Consiste en el abandono del activismo 
dentro la vida de las instituciones de educación superior, del ejercicio básico del gobierno compartido, o si se prefiere, de la participación de los estudiantes y los académicos en la vida de las instituciones para hacer de ellas una comunidad viva más allá de un conglomerado de personas, facultades e institutos puestos por determinación histórica en el mismo lugar buscando la excelencia -lo que sea que eso signifique-.

Esto debe entenderse literalmente. La pobre construcción de comunidad en las universidades públicas no es el resultado de un ejercicio deliberado, no es el resultado de una decisión y no es exactamente el resultado de la indiferencia. Me parece que es una suerte de "efecto de despolitización" que emerge de las tendencias y de las políticas institucionales propias de los discursos seductores de la era del conocimiento, previamente abordados. Políticas, regulaciones, dispositivo híbrido que han conseguido ocupar a estudiantes y académicos en una vida de producción intensa, llena de evaluaciones, guiada por indicadores de productividad, administrada por calendarios llenos de actividades, orientada hacia una cierta idea de calidad que ha puesto en suspenso, y casi ha ocultado el hecho de que una de las responsabilidades de ese gran colectivo es la participación activa en el cogobierno de las instituciones universitarias.

No quiero ser injusto, pues en muchas universidades incluida la Veracruzana, los estudiantes eventualmente reclaman por diferentes cosas como las condiciones en las aulas, por el trato que reciben de los profesores e, inclusive, reclaman contra los gobiernos locales desde la plataforma universitaria por asuntos como la violencia. En la Universidad Veracruzana recientemente se verificó una gran movilización cuando del gobierno del estado comenzó a condicionar la entrega del financiamiento a la universidad poniendo en riesgo su existencia. Pero aunque este tipo de acciones son importantes casi siempre son episódicas, de baja intensidad, y muchos casos son convocadas o lideradas por estructuras universitarias que asumen la dirección de la protesta.

Lo que estaría faltando -desde mi punto de vista- es lo que Jaques Rancière (1996) ha nombrado como el desacuerdo, el litigio por la distribución de los lugares sociales y por la forma en que se ha llegado a dicha distribución. Por ejemplo, hoy en día es más que evidente que las instituciones de educación superior incluidas las universidades están intentando adaptarse a las exigencias de un entorno económico cambiante y adverso en más de un sentido. Dicho entorno no ofrece grandes alternativas laborales para miles de estudiantes que ingresan y egresan de las universidades. En general se observa una precarización del empleo y una marcada diferenciación social donde los altos ingresos y los beneficios del desarrollo han tendido a acumularse en unas cuantas manos, dejando muy poco para "el resto". En este sentido el título universitario no asegura lo que durante mucho tiempo se ha conocido como movilidad social ascendente.

Es paradójico cómo se carga a la universidad con esta responsabilidad cuando claramente ninguna institución de educación superior puede cambiar las dinámicas del entorno por sí sola. Los estudiantes, en cambio, son preparados y sometidos a diferentes esquemas de formación flexible que constantemente se actualiza en términos de los conocimientos y los contenidos, pero que ultimadamente no puede "responder" cabalmente a ese entorno que los estudiantes enfrentarán cuando sean egresados.

Llama mi atención que muy pocos académicos y estudiantes han puesto en cuestión esa forma de organización de lo social. Llama mi atención que las condiciones de empleabilidad y desarrollo profesional y personal tan adversas para los estudiantes y para miles de académicos no están en el centro de algún proceso de politización emanados desde las universidades. En México y en otras partes de América Latina las instituciones educativas, en particular de carácter público, habían asumido como uno de sus grandes compromisos funcionar como caja de resonancia de los problemas 
colectivos. Habían asumido el compromiso de revisar críticamente las condiciones de existencia de nuestras sociedades y la forma en que llegamos a ellas.

Al parecer esta capacidad de análisis y de devolución de la crítica está fuertemente contenida, aunque no desaparecida, por un efectivo dispositivo híbrido de gestión del pensamiento y de la acción académica: en la era del conocimiento, arropados por nuevas narrativas sobre cómo producir, utilizar, gestionar o vender conocimiento, la pregunta por nuestro lugar en la distribución de las partes sociales, parece estar extraviada. Los efectos de despolitización no nacen en la universidad, sino que son el resultado de un agregado de procesos sociales que, sin embargo, no son puestos en cuestión de manera sistemática en nuestras aulas.

Sin duda nuestras universidades podrían beneficiarse de un proceso desacuerdo como el que se dio hace más de cien años en la Universidad de Córdoba, un proceso que empieza por lo más elemental, por preguntarse acerca de cómo nos organizamos, cómo asumimos nuestra responsabilidad social, acerca de quiénes tienen derecho a utilizar la voz en los espacios universitarios y en qué circunstancias, acerca de la prioridad que se ha otorgado al cumplimiento de políticas de gestión del conocimiento y de la formación que están orientadas a un entorno social político y económico que ofrece condiciones de precarización y exclusión social crecientes.

La puesta en cuestión de la que estoy hablando no se procesa solamente a través de nuevas políticas educativas o de nuevos procesos de gestión del cambio. En general, las instituciones de educación superior como las universidades se han vuelto expertas en resolver problemas a través de los paradigmas de administración o de gestión educativa. Este es posiblemente uno de los mayores aprendizajes que se han derivado de la influencia de las organizaciones internacionales en el diseño institucional y en el gobierno universitario: su capacidad para ser gestoras del desacuerdo y del cambio en sus propios términos.

La puesta en cuestión a que me refiero, en cambio, implica revisar de manera colectiva y litigiosa en términos epistemológicos y también políticos, el tipo de conocimiento que se está produciendo, que se recrea a través del currículum, que se orienta a patentes, a certificaciones y que parece estar convirtiendo a las universidades públicas en fábricas de conocimiento lideradas, en ocasiones por príncipes, en ocasiones por burócratas y en ocasiones por gerentes (Acosta, 2009) "bien intencionados", que buscan asegurar el futuro de las instituciones mientras ponen riesgo el cumplimiento de sus más elementales responsabilidades. $Y$ donde los académicos parecen estarse constituyendo en una estructura social con una élite de ingresos garantizados, con accesos a apoyos y financiamientos mientras "el resto" conforma una gran base de académicos en precarización.

\section{Cierre: la reforma como imaginario}

Las reformas educativas dan cuenta procesos de ruptura y también de continuidad. A partir de un proceso de reforma hay una suerte de rompimiento en el orden simbólico prevaleciente y se instaura un nuevo imaginario. Dicho imaginario puede ayudar a procesar los cambios, pues ofrece posibilidades, pero en la medida en que se va consolidando varios de los elementos que configuraron el imaginario de inicio, van cambiando. Algunos se pierden en la nube de los tiempos y otros se activan constantemente, enriqueciéndose mientras circulan produciendo una nueva simiente. A partir de 1918, las universidades públicas en gran parte América Latina y en México se han venido transformando a la luz de una reserva simbólica que se configuró como 
imaginario habitado por nociones de responsabilidad social, de apertura, y gobierno compartido en un marco de autonomía.

Pero la autonomía es relativa y ha cambiado en función de las condiciones históricas de cada país, de cada institución. Desde condiciones de dictadura, hasta de pobreza o de violencia generalizada, las universidades en tanto comunidades de diversos, han experimentado de todo, y en el último ciclo de cambio, inaugurado con el ascenso de las tecnologías digitales, la globalización y el neoliberalismo han entrado en una fase que hace muy difícil de compararlas con las del siglo pasado, sea en términos de diseño, de organización, de gobierno o de misión.

Los procesos de diversificación de la educación superior han ayudado de una forma u otra a que más personas tengan posibilidad de educarse y de tener una vida personal y profesional enriquecida en muchos sentidos. Las políticas educativas que resumí al inicio del texto han generado que las universidades públicas en México formen un sistema sofisticado, pero también han llevado a la ausencia o la precarización de vínculos, de vasos comunicantes entre estudiantes, profesores, instituciones y programas académicos.

He intentado mostrar los cambios en el caso mexicano recorriendo resumidamente los rasgos del sistema de educación superior en el siglo que ha pasado desde la Reforma Universitaria de Córdoba. He mostrado rasgos de la nueva narrativa del conocimiento con énfasis en las dinámicas de despolitización en años recientes, que ha llevado a la emergencia de un paradigma de gestión protogerencialista. He señalado que la narrativa sobre el conocimiento no es una de tipo impositivo, es decir, no obliga, es inclusiva, está compuesta por significantes con gran capacidad de vaciamiento y ha sido reapropiada por diferentes actores políticos y económicos, quienes han contribuido a generar diferentes discursos con gran capacidad de convencimiento ya que prometen salvarnos del atraso y de la ignorancia, de la pobreza y el desempleo, de la "irrelevancia" de la educación universitaria. Son discursos que operan al interior de un dispositivo híbrido que promueve la "competitividad", la "productividad", la "calidad", y acarrea un efecto de despolitización.

El argumento elemental de este escrito se reitera, entonces: las universidades públicas de México, como otras de América Latina se están transformando en entidades híbridas, en la tensión de responder a los preceptos de autonomía de gobierno y gestión, la presión de los gobiernos y las tendencias impulsadas por agentes externos que poco a poco han introducido nuevas racionalidades que hacen sistema con discursos de igualdad, equidad y responsabilidad social. Este hibridismo tiene diferentes efectos despolitizantes que deben ser desmotados, pues tendrán consecuencias drásticas para la idea de universidad pública de orientación científica y de compromiso social que se ha intentado construir durante varias décadas.

Se extraña un nuevo proceso de politización que no necesariamente pase por las juntas de gobierno, las asociaciones de egresados o de estudiantes gestionadas internamente o ultimadamente por los partidos políticos que todavía logran filtrarse dentro de las instituciones de educación superior. Se extraña, y por tanto es necesario, un proceso de revisión del gobierno y de la vida de las universidades públicas mexicanas porque las narrativas de la era del conocimiento han modificado radicalmente el orden simbólico desde el cual las imaginamos. El aniversario del movimiento de reforma de Córdoba es un momento idóneo para ello, es la oportunidad de todo un continente para muchas generaciones por venir. 


\section{Referencias bibliográficas}

» Acevedo, A. (2011). A cien años de la reforma de Córdoba, 1918-2018. La época, los acontecimientos, el legado. Historia y Espacio, 7(36).

» Acosta, A. (2009). Príncipes, burócratas y gerentes. El gobierno de las universidades públicas en México. Ciudad de México, México: ANUIES.

» Buchbinder, P. (2018). Pensar la Reforma Universitaria cien años después. Revista Iberoamericana de Educación Superior, IX(25), pp. 86-95.

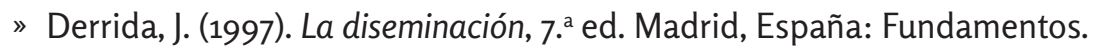

»Drucker, P. (1969). The Age of Discontinuity: Guidelines to Our Changing Society. New York, EE.UU.: Harper and Row.

" Hayek, F. (1937). Economics and Knowledge, Presidential Address delivered before the London Economic Club. Economica, (IV), pp. 33-54.

"Laclau, E. (2014). Los fundamentos retóricos de la sociedad. Buenos Aires, Argentina: Fondo de Cultura Económica.

» Lyotard, J. F. (1981). La condición postmoderna, Madrid, España: Cátedra.

" Machlup, F. (1962). The Production and Distribution of Knowledge in the United States. Princeton, New Jersey, EE.UU.: Princeton University.

" Marsiske, R. (coord.). (1999). Movimientos estudiantiles en la historia de América Latina. Ciudad de México,México: CESU - UNAM.

$»$ (coord.). (2015). Movimientos estudiantiles en la historia de América Latina. Ciudad de México, México: CESU - UNAM.

» Marván, I. (2017). Cómo hicieron la Constitución de 1917. Ciudad de México, México: Centro de Investigación y Docencia Económicas, A.C.

»Miller, P. y Rose, N. (2008). Governing the Present: Administering Economic, Social and Personal Life. Cambridge, United Kingdom: Polity.

» OCDE. (1996). The Knowledge-Based Economy. París, Francia: Organización para la Cooperación y el Desarrollo Económico.

"_. (2000). Knowledge Management in the Learning Society. Paris: Organización para la Cooperación y el Desarrollo Económico.

»Popkewitz, T. (2007). Cosmopolitanism and the Age of School Reform: Science, Education, and Making Society by Making the Child. New York, EE.UU.: Routledge.

» Rancière, J. (1996). El desacuerdo. Filosofía y política. Buenos Aires, Argentina: Nueva Visión.

" Rodríguez, R. (1995). Expansión del sistema educativo superior en México 1970-1995. En M. Fresán Orozco (ed.), Tres décadas de políticas del Estado en la educación superior, pp. 167-205. Ciudad de México, México: ANUIES.

»Saur, D. y Servetto, A. (coords.). (2013). Universidad Nacional de Córdoba. Cuatrocientos años de historia. Córdoba, Argentina: UNC.

»SEP (Secretaría de Educación Pública) (2001). Programa de Nacional de Educación. Ciudad de México, México: Secretaría de Educación Pública. 
»_. (2007). Programa Sectorial de Educación. Ciudad de México, México: Secretaría de Educación Pública.

"_. (2013). Programa Sectorial de Educacion. Ciudad de México, México: Secretaría de Educación Pública.

»SEP y CONACYT (Consejo Nacional de Ciencia y Tecnología) (1996). Programa de Mejoramiento del Profesorado. Ciudad de México, México: Secretaría de Educación Pública y Consejo Nacional de Ciencia y Tecnología.

»Sther, N. (2004). The Governance of Knowledge, New Brunswick, EE.UU.: Transaction.

» Treviño, E. (2015). La educación superior y el advenimiento de la sociedad del conocimiento. Equivalencias y diferencias en los discursos y políticas de transformación educativa en los ámbitos nacional e internacional. Ciudad de México, México: ANUIES.

» Treviño, E., Galindo, J. y Ducey, M. (coords.). (2018). Cien años de la Constitución de 1917. Análisis interdisciplinarios. Xalapa, México: Universidad Veracruzana.

" Touraine, A. (1969). La société post-industrielle. Naissance d une société. París, Francia: Denoël.

» Tünnermann, C. (2008). Noventa años de la Reforma Universitaria de Córdoba (1918-2008). Buenos Aires, Argentina: CLACSO.

"Unesco (1998). Declaración mundial sobre la educación superior para el Siglo XXI: visión y acción, y Marco de acción prioritaria para el cambio y el desarrollo de la educación superior adoptados por la Conferencia Mundial sobre la educación superior: visión y acción. París, Francia.

$\gg$ . (2005). Hacia las sociedades de conocimiento. París, Francia.

"Universidad Veracruzana. (1998). Consolidación y proyección hacia el Siglo XXI, Universidad Veracruzana, 1998-2001. Veracruz, México.

\section{Ernesto Treviño Ronzón}

Doctor en Ciencias por el Centro de Investigación y de Estudios Avanzados del Instituto Politécnico Nacional (CINVESTAV-IPN); investigador titular en el Instituto de Investigaciones Histórico-Sociales de la Universidad Veracruzana, México; Miembro del Sistema Nacional de Investigadores. Correo electrónico: etrevino@uv.mx 
